Pensamiento Crítico No. 7, pág.. 21 - 33

\title{
Financiamiento de Programas Sociales El Caso del Programa de Comedores Populares en el Perú
}

César A. Sanabria Montañez

Resumen: El presente artículo explica el rol que juegan las organizaciones sociales en el financiamiento de los Programas Sociales, como es el caso del Programa de Comedores Populares en el Perú, donde el financiamiento recae fundamentalmente en la población (asociaciones de mujeres) que gestiona dichos Comedores Populares, pues sus aportes, mediante el trabajo y el "pago de bolsillo", representan la mayor parte de recursos (cerca del $74 \%$ de recursos necesarios para que funcione el Programa). Por lo que en el presente artículo se presenta de manera explícita esta forma de organización como breve reseña del modelo de gestión y financiamiento de los Comedores Populares.

Palabras claves : Programas sociales, comedores populares, política social, financiamiento, alimentación complementaria. 


\section{César A. Sanabria Montañez}

\section{Antecedentes}

Los Comedores Populares en el Perú nacen a raíz de la crisis económica de los años 70 y 80 , en el Perú, una de sus características más importantes es que está conformado por mujeres de los sectores pobres, quienes de modo autogestionario formaron Clubes de madres o también llamados Comedores Populares, organización que les permite adquirir y preparar alimentos, enfrentaron inicialmente solas, la inflación, el desempleo, el hambre y la enfermedad en sus primeros años de vida. El Estado recién empieza a apoyar estas iniciativas en los años 80, y ya en los años 90 este apoyo se hace más extensivo y se convierte en el Programa de Comedores Populares, que continúa hasta la actualidad.

La interacción entre Estado y Comedores Populares se da a través del liderazgo estatal, donde existen dos productos, a) la entrega de alimentos y b) la entrega de dinero (subsidio económico) a Organizaciones Sociales de Base. La actuación del Estado se da a través del Programa Nacional de Apoyo Alimentario (PRONAA), organismo que pertenece al Ministerio de la Mujer y Desarrollo Social (MIMDES), y también dentro del proceso de descentralización, donde participan las Municipalidades Provinciales acreditadas a las cuales se han trasladado las funciones de dirección, organización, supervisión, control y entrega de alimentos. En términos generales la actuación del Estado y su interacción con las organizaciones de base se destaca en el gráfico $\mathrm{N}^{\circ} 1$ y gráfico $\mathrm{N}^{\circ} 2$.

\section{Gráfico No 1}

\section{Interacción entre el Estado y Organizaciones de Base}

\section{En el Programa de Comedores Populares}

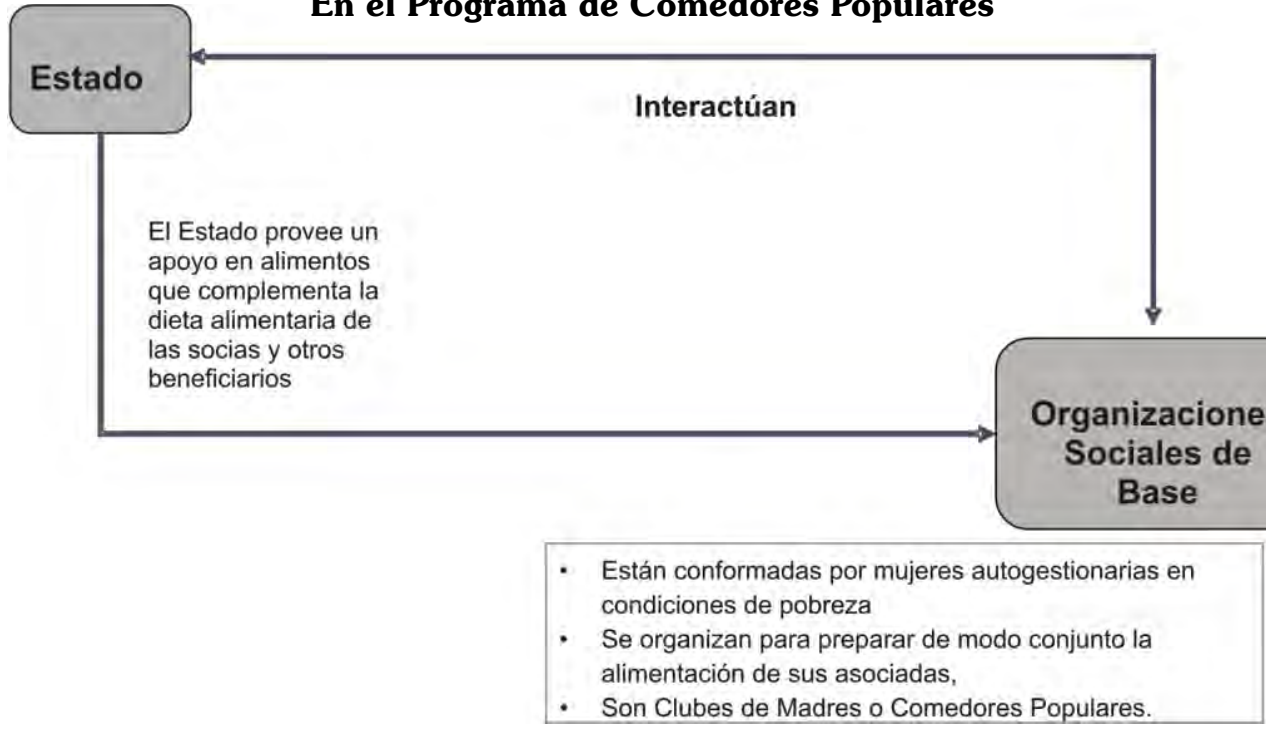


Financiamiento de programas sociales: el caso del programa de comedores populares en el Perú

\section{Gráfico $\mathbf{N}^{\circ} 2$}

\section{Productos del Programa de Comedores Populares}

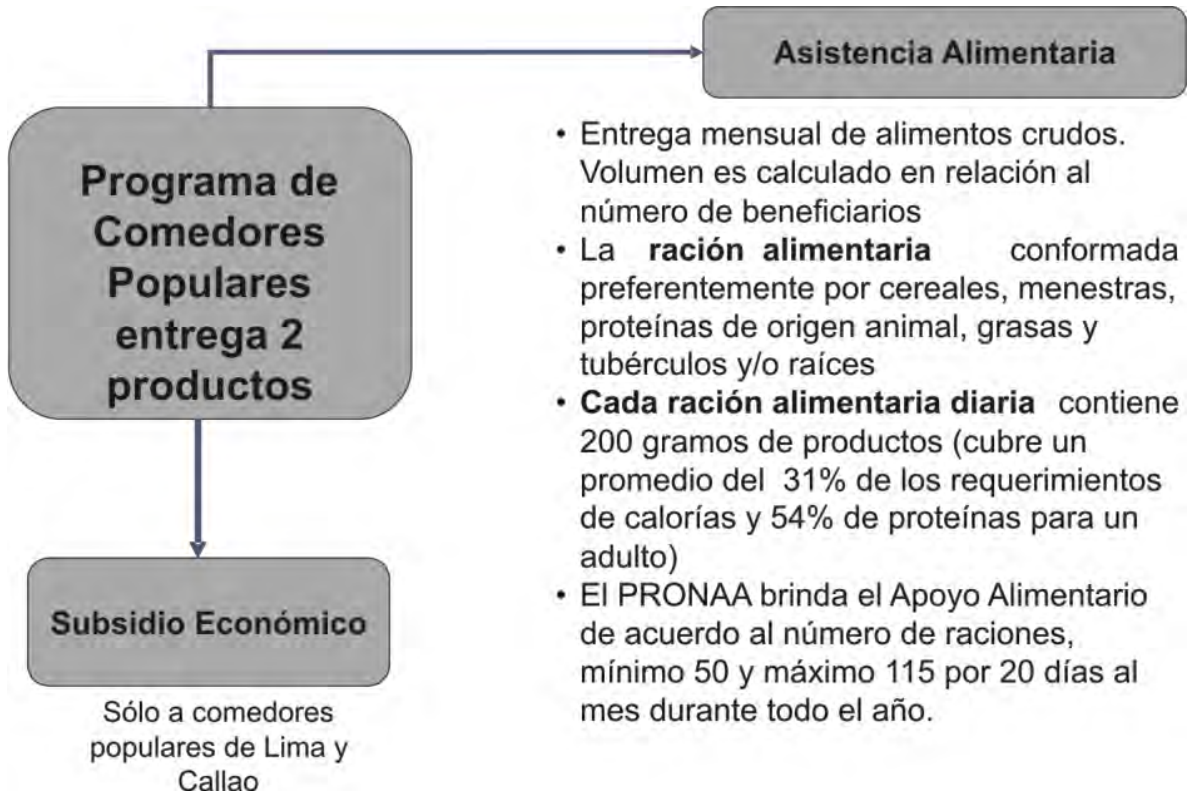

El objetivo del Programa de Comedores Populares es mejorar las condiciones de acceso a la alimentación de personas de bajos recursos, constituyéndose los comedores en agentes de cambio, con carácter comunitario, desempeño dinámico y desarrollo organizacional ${ }^{1}$.

Hay que señalar que el Programa de Comedores se encuentra inmerso dentro del proceso de descentralización, así el PRONAA, desde el año 2003 y en el marco de la normatividad dada por el Decreto Supremo Nº 036-2003-PCM y el Decreto Supremo Nº 088-2003-PCM, dio inició al Proceso de Transferencia a los Gobiernos Locales de los Programas de Complementación Alimentaria. Dicha acción también se realizó en virtud a la Segunda Disposición Complementaria de la Ley de Bases de la Descentralización $\mathrm{N}^{\circ} 27783^{2}$. Para la transferencia de Programas a los gobiernos locales (Municipios Provinciales), estas normas señalan que estos Municipios hayan sido acreditados previamente por el Consejo Nacional de Descentralización. La transferencia en la actualidad viene dándose de manera paulatina, identificándose una variedad de limitaciones. Para el año 2005 se habían transferido los Programas de Complementación Alimentaria a 125 gobiernos Bócales provinciales . 


\section{César A. Sanabria Montañez}

Para el Caso de Lima Metropolitana, se ha constituido una Comisión Especial encargada de proponer la modalidad de transferencia y el modelo de gestión descentralizada del Programa de Complementación Alimentaria ${ }^{4}$ y que a la fecha este proceso está estancado, lo cual significa que $60 \%$ del total de Comedores Populares en el Perú (ubicados en Lima Metropolitana) aún no han sido transferidos y que continúan bajo la organización del PRONAA.

En este estado de situación, el modelo de financiamiento de Programa de Comedores Populares que buscamos analizar, tiene en el modelo de gestión y organización del Programa dos actores importantes el Estado y las Organizaciones sociales de base, lo cual implica dos fuentes de financiamiento.

El financiamiento público, basado en recursos provenientes de impuestos o préstamos de organismos multilaterales que obtiene el Estado y que destina para el funcionamiento de los Comedores Populares, y el financiamiento privado a cargo de la comunidad: las integrantes de las Organizaciones Sociales de Base y los beneficiarios que consumen los menús del comedor popular, este financiamiento proviene entonces de: a) los aportes vía pago de bolsillo, pago por el menú que realizan los consumidores por el menú, b) aportes en trabajo que realizan las socias de las organizaciones sociales de base y $\mathbf{c}$ ) aportes a través de actividades que hacen las socias para cubrir los gastos de los "casos sociales".

El modelo financiamiento que se señala se observa en el esquema presentado en el gráfico $\mathrm{N}^{\circ} 3$.

\section{Gráfico No 3 .}

\section{Modelo de financiamiento del Programa de Comedores Populares}
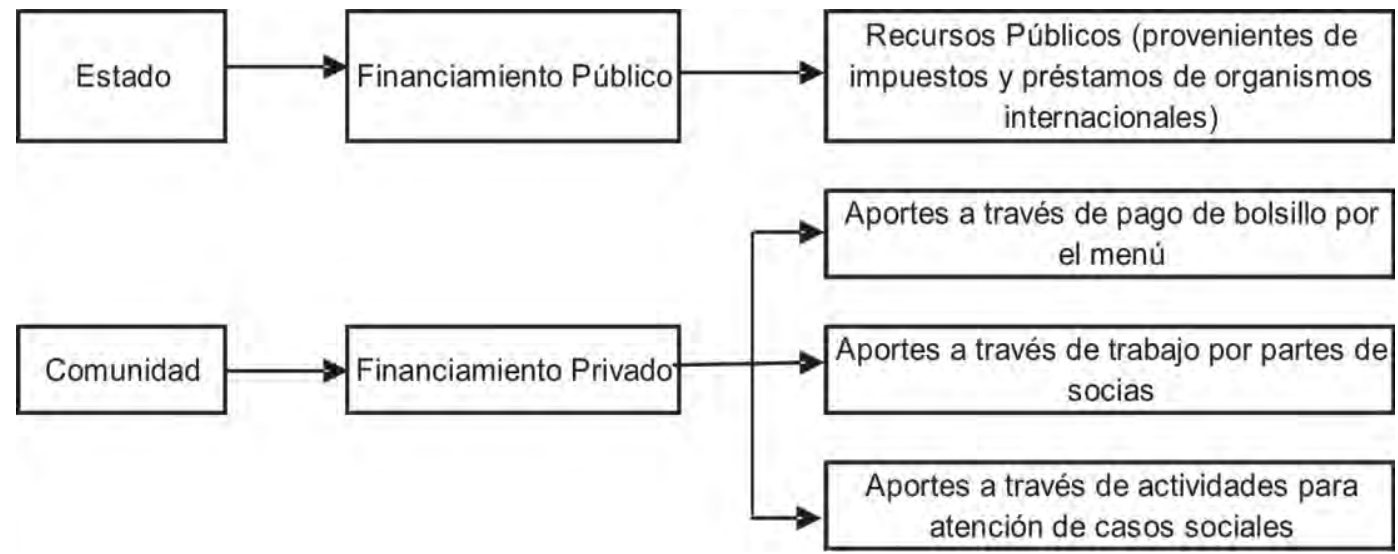
Financiamiento de programas sociales: el caso del programa de comedores populares en el Perú

Este modelo señala la necesaria participación en el financiamiento de la comunidad organizada para la existencia de los Comedores Populares y que no todos recursos provienen del Estado; asimismo hace necesario la interacción del Estado y de la comunidad para lograr los objetivos del Programa. Por otra parte, el financiamiento no necesariamente se refleja en términos de flujo monetario, sino que existen aportes de recursos implícitos, como es el trabajo que realizan las socias en el comedor y que se estimó en el estudio.

\section{Material y métodos}

El estudio que presentamos sólo incluye a los Comedores Populares dentro de la responsabilidad del PRONAA, es decir de Comedores Populares que no han sido transferidos a los Gobiernos Locales, para ello se definió una muestra a nivel nacional definida en 4 dominios, Lima Metropolitana, Resto Costa, Sierra y Selva. Ver para ello el Cuadro $N^{\circ} 1$. Se seleccionó una muestra probabilística, polietápica, independiente por cada dominio de estudio y con probabilidades proporcionales al tamaño, con un intervalo de confianza del 95\%, un margen de error del $7 \%$ y una potencia de prueba de hipótesis del $90 \%$.

Para el caso del estudio de gestión y propiamente del financiamiento de los Comedores Populares la unidad de análisis fue el Comedor Popular.

\section{Cuadro $\mathbf{N}^{\circ} 1$}

\section{Distribución de la muestra por unidades de muestreo, dominios y grupos}

\begin{tabular}{|l|c|c|c|c|c|c|c|c|c|c|}
\hline \multirow{2}{*}{ Unidad de muestreo } & \multicolumn{2}{|c|}{ Lima Met. } & \multicolumn{2}{|c|}{ R. Costa } & \multicolumn{2}{c|}{ Sierra } & \multicolumn{2}{c|}{ Selva } & \multicolumn{2}{c|}{ Total } \\
\cline { 2 - 14 } & P & E & P & E & P & E & P & E & P & E \\
\hline $\mathrm{N}^{\circ}$ Comedores & 50 & 62 & 50 & 50 & 60 & 60 & 30 & 30 & 190 & 202 \\
\hline $\mathrm{N}^{\circ}$ Distritos & 11 & 10 & 11 & 11 & 10 & 12 & 5 & 6 & 39 & 38 \\
\hline $\mathrm{N}^{\circ}$ provincias & 2 & 2 & 5 & 5 & 6 & 6 & 3 & 3 & 16 & 16 \\
\hline
\end{tabular}

$\mathbf{P}=$ Planificado $\mathbf{E}=$ Ejecutado

La evaluación de gestión y financiamiento del programa se hizo partiendo del modelo que se muestra en el Gráfico $\mathrm{N}^{\circ} 4$, en el sentido de examinar los recursos que destina la Alta Dirección al Programa y la actuación de los actores en la gestión operativa, ello a partir de un cuestionario semi-estructurado se evaluó la participación de todos los actores del Programa Nacional de Comedores Populares en los diferentes niveles de gestión. De esta manera se diseñaron diversas entrevistas a funcionarios del PRONAA y se revisaron los respectivos documentos de gestión. 


\section{César A. Sanabria Montañez}

Los indicadores de gestión para evaluar el financiamiento del Programa de Comedores Populares fueron los siguientes:

- Grado de organización del Programa en sus diferentes niveles.

- Eficacia del Programa: programación y ejecución de actividades del 2004, criterios de selección de los intervenidos, raciones programadas vs. distribuidas.

- Eficiencia del Programa: programación y ejecución presupuestal, mecanismos de transferencia de recursos a los intervenidos, logística alimentaria.

- Porcentaje de madres beneficiarias y responsables de la preparación de alimentos capacitadas.

- Grado y formas de participación de madres beneficiarias en los Comedores Populares.

- Número de días de atención del Comedor Popular al año y horarios de atención diaria.

- Pago por menú según tipo de beneficiario

- Formas y Actividades realizadas para la atención de los casos sociales

\section{Gráfico $N^{\circ} 4$ : Niveles de Evaluación de la Gestión del Programa}

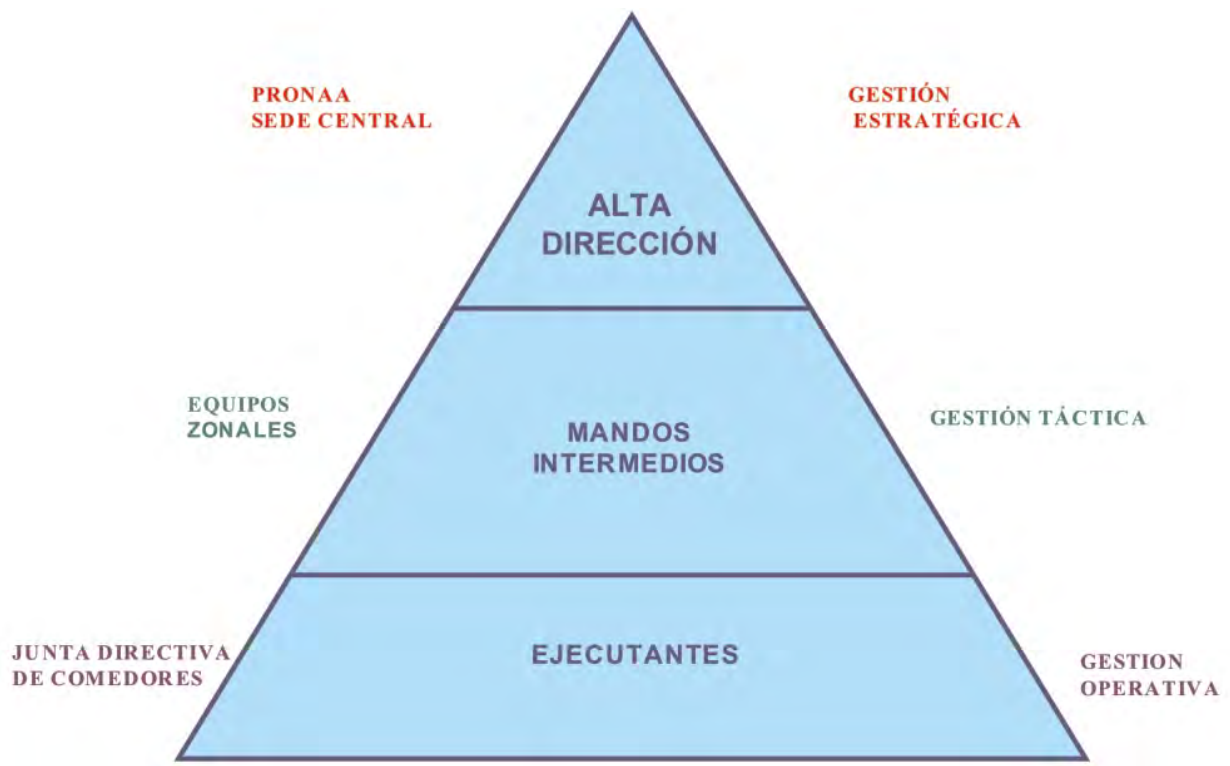

El avance en el proceso de descentralización se investigó a través de entrevistas con funcionarios de los Equipos Zonales, información del Equipo Central del PRONAA y también 
Financiamiento de programas sociales: el caso del programa de comedores populares en el Perú

pidiéndoles la opinión de los representantes de la Junta Directiva de los Comedores sobre la descentralización. Las preguntas a plantearse se incluyeron en el formulario de la encuesta de gestión.

\section{Fuentes de financiamiento del Programa de Comedores Populares}

El programa de Comedores Populares se financia por cuatro fuentes:

a) El financiamiento público (transferencias, donaciones y recursos ordinarios que administra el PRONAA).

b) El pago que realizan las socias y intervenidos de los Comedores Populares.

c) El trabajo que realizan las socias y beneficiarios en la preparación de los menús y funcionamiento del comedor popular.

d) Las actividades que realizan las socias para cubrir la dieta de los llamados casos sociales.

Respecto del financiamiento público, los montos de metas físicas destinados a atención de Comedores Populares, desde el aÑo 2003 ha ido disminuyendo, ello en razón del proceso de transferencia a gobiernos locales de los programas de complementación alimentaria en la que está inmerso el programa de Comedores Populares. Ver Cuadro $\mathbf{N}^{\circ} 2$.

En cuanto a niveles de eficiencia en las metas ejecutadas respecto de las programadas, vemos que hay un alto nivel de cumplimiento. En el año 2004 se presenta un mejor nivel de cumplimiento, llegando a cubrir al $101 \%$ de intervenidos y realizar un $105,3 \%$ de las inversiones programadas.

También como fuente de financiamiento del Estado está el subsidio monetario directo, el PRONAA a través del Equipo Zonal Callao ${ }^{6}$ proporciona subsidio pecuniario a grupos de mujeres organizadas en comedores o clubes de madres de Lima y Callao, con la finalidad de cubrir parte del costo de una ración alimentaria diaria y contribuir a elevar el nivel alimentario de la población que se encuentra en extrema pobreza. Ver Cuadro $\mathbf{N}^{\circ} 3$.

El subsidio es una ayuda en efectivo que reciben algunos los Comedores Populares de Lima y Callao, para financiar la compra de productos perecibles para la preparación de raciones de alimentos durante 300 días al año. El monto del subsidio es de 0.25 nuevos soles por ración. Este subsidio económico es adicional a la entrega de raciones en producto.

Esta actividad se financia con recursos ordinarios, habiéndose ejecutado en el año 2003 un total de S/. 9,451,615 que representó el 99.49\% de la meta programada. Con este monto se logró atender a 170,950 personas. 
César A. Sanabria Montañez

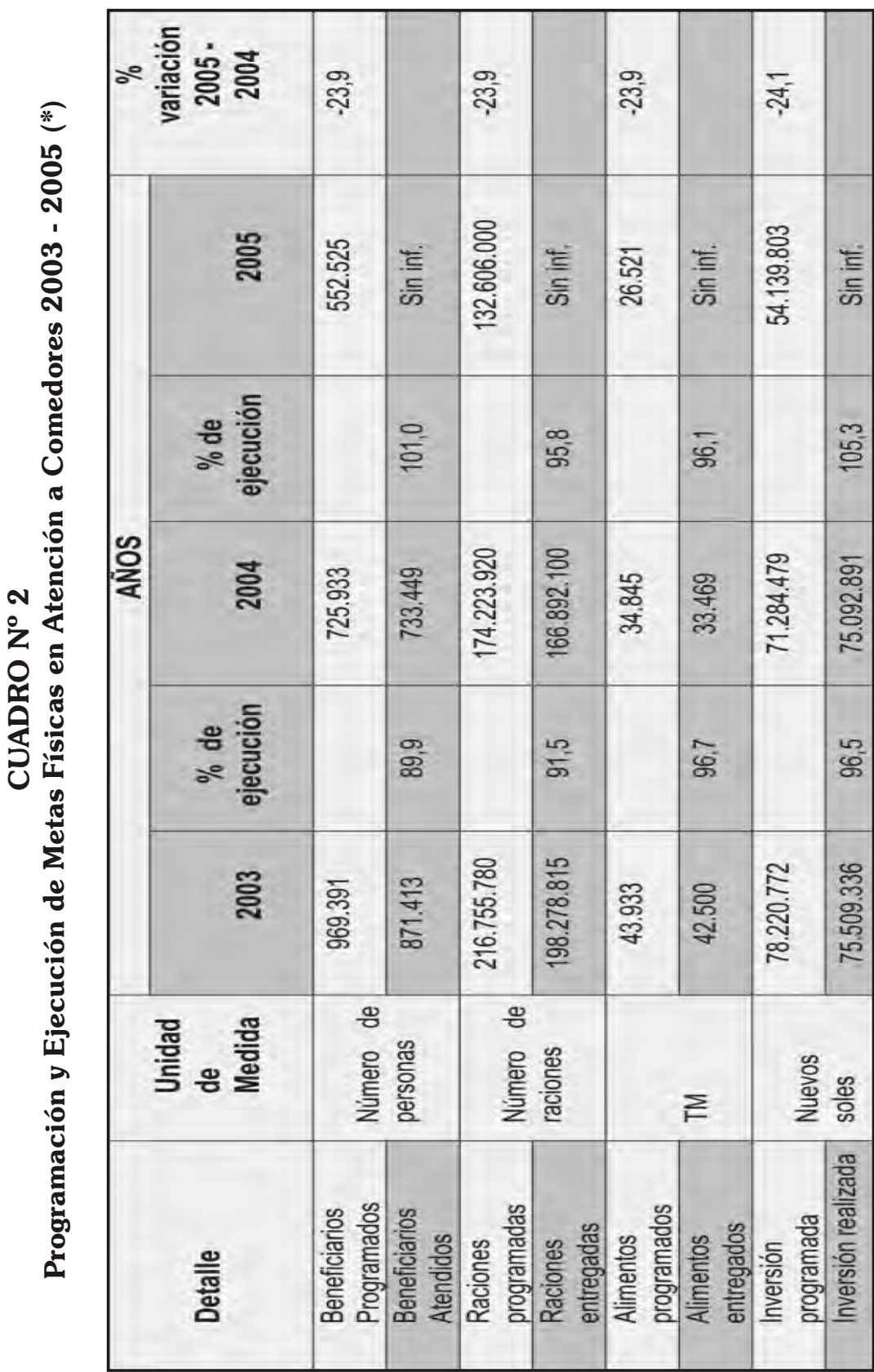

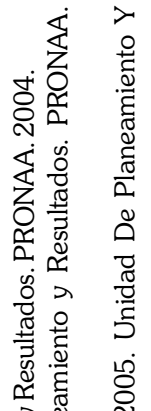

रे

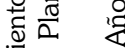

है च

뜬

可

흉 용

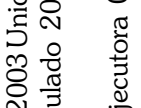

हु छ

究

은 즈

宽 을 움

气

중

요

ปั้

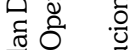

힐

중 क्ष

$<$ 政

:

䒕 岃员远肯 के山ें

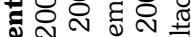
要,

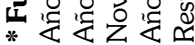


Financiamiento de programas sociales: el caso del programa de comedores populares en el Perú

Para el año 2005 se programó continuar con subsidios monetarios a los comedores de Lima y Callao por 240 días/año, equivalente a 9'482,268 nuevos soles. Se estimó que estos recursos beneficiarán aproximadamente a 158,038 personas.

El21.8\% de la muestra de Comedores de Lima - Callao recibe este subsidio.

\section{Cuadro No 3: Presupuesto Programado y Ejecutado de Subsidios Monetarios a Comedores Populares de Lima - Callao (*)}

\begin{tabular}{|c|c|c|c|c|}
\hline \multirow{2}{*}{ Año } & \multicolumn{3}{|c|}{ Presupuesto } & \multirow{2}{*}{ Beneficiarios } \\
\cline { 2 - 4 } & Programado & Ejecutado & $\begin{array}{l}\text { Porcentaje } \\
\text { ejecución }\end{array}$ & Bencion \\
\hline 2003 & $9,500,065$ & $9,451,615$ & 99.49 & 170,950 \\
\hline 2004 & $9,482,280$ & $9,526,423$ & 100.47 & s.i. \\
\hline 2005 & $9,482,268$ & s.i. & - & 158,038 \\
\hline
\end{tabular}

En Nuevos Soles Corrientes.

(*) Fuentes: Año 2003: Evaluación Anual del Plan De Trabajo Institucional-PTI 2003 Unidad de Planeamiento y Resultados. PRONAA. 2004.

Año 2004: Evaluación del Plan Operativo Institucional Reformulado 2004. Unidad de Planeamiento y Resultados. PRONAA. Noviembre 2005.

Año 2005: Plan Operativo Institucional Reformulado Unidad Ejecutora 005 PRONAA. Año 2005. Unidad De Planeamiento Y Resultados. Lima, Diciembre 2005.

La segunda fuente de financiamiento del programa de Comedores Populares es el pago que realizan las socias y intervenidos del Programa por el menú diario que se prepara en los comedores y se expende al público y socias. Hay que anotar que esta modalidad, es una forma importante de lograr los objetivos del Programa, ya que según las normas, el mínimo de socias activas que debe tener un comedor es de 15, y el mínimo de raciones a preparar es de 50 en caso de ubicarse en zonas urbanas y producir los menús diarios de 5 días a la semana; en caso de ubicarse en la zona rural, el mínimo de atención proyectada es de 3 veces por semana y las raciones no deben ser menores a 80 diarias?.

Se destaca que en los Comedores Populares las socias que participan en la cocina (preparación del menú) y el funcionamiento del Comedor están exceptuadas generalmente del pago del menú, en cambio las otras socias y otros usuarios sí pagan por el menú diario. Hay distintas tarifas y formas de pago en los Comedores Populares en el Perú. En el Cuadro No 4 , se presentan los precios promedio pagados por los usuarios del comedor por menú diario según dominio, habiendo un pago diferenciado según sea socia o no del Comedor Popular. Como se puede ver, en la sierra los promedio de precios pagados son los más baratos y en Lima los más caros. En promedio en el Perú cada beneficiario financia de modo directo (pago de bolsillo) el Programa de Comedores con 0,8 nuevos soles por ración si es socia del comedor y 0,9 nuevos soles si no es socia del comedor. 
César A. Sanabria Montañez

Cuadro $\mathbf{N}^{\circ} 4$

Pago Promedio por Menú Diario que Realizan los Usuarios de los Comedores Populares Según Dominio. (en nuevos soles )

\begin{tabular}{|l|c|c|c|r|r|}
\hline \multicolumn{1}{|c|}{ Detalle } & Nacional & Lima & $\begin{array}{r}\text { Resto } \\
\text { Costa }\end{array}$ & Sierra & \multicolumn{1}{|c|}{ Selva } \\
\hline Socias del comedor & 0.8 & 1.1 & 0.9 & 0.3 & 0.7 \\
\hline No socias del comedor & 0.9 & 1.4 & 1.0 & 0.4 & 0.8 \\
\hline
\end{tabular}

Fuente: Encuesta de Gestión a Junta Directivas de Comedores Populares.

Si tomamos como referencia estos precios pagados por los usuarios por el menú diario, el financiamiento (pago de bolsillo) total de las socias sería de 112.159.467 nuevos soles y de las no socias 23.084.344 nuevos soles, haciendo un total de 135.243.812 nuevos soles para el año 2003. El año 2004 el financiamiento (pago directo de bolsillo) de las socias se estima en 94.405.088 nuevos soles y de las no socias se estima en 19.430.188 nuevos soles, haciendo un total de 113.835.277 nuevos soles de aporte de los usuarios para el funcionamiento del programa.

Una tercera fuente de financiamiento del Programa de Comedores Populares esta dada por el trabajo que realizan las socias en la preparación de los menús. Este aporte se ha estimado para el año 2005 en base a tres parámetros: el número promedio de socias dedicadas a la preparación de raciones, el número de días de funcionamiento de los Comedores Populares al mes, la distribución de comedores según el tiempo de trabajo diario dedicado al comedor por las socias y la remuneración mínima vital a esa fecha ${ }^{9}$. Este financiamiento se ha estimado en una suma total de 95,896,032 nuevos soles para el año 2005. Ver Cuadros 5, 6y 7.

\section{Cuadro $\mathbf{N}^{\circ} 5$}

\section{Promedio de Socias que Trabajan en el Comedor y Promedio de Días que Funcionan los Mismos, por Dominios}

\begin{tabular}{|l|c|c|c|c|}
\hline \multicolumn{1}{|c|}{ Información } & Lima & R. Costa & Sierra & Selva \\
\hline $\begin{array}{l}\text { Promedio de socias que } \\
\text { labora en el comedor }\end{array}$ & 3.3 & 2.1 & 2.7 & 2.2 \\
\hline $\begin{array}{l}\text { Promedio de días que } \\
\text { funciona el comedor }\end{array}$ & 23.0 & 21.4 & 15.1 & 17.1 \\
\hline
\end{tabular}

Fuente: Encuesta de Gestión a Junta Directivas de Comedores Populares 
Financiamiento de programas sociales: el caso del programa de comedores populares en el Perú

\section{Cuadro $\mathrm{N}^{\circ} 6$}

\section{Distribución de Comedores Según Días de Trabajo de Socias, por Dominios}

\begin{tabular}{|c|c|c|c|c|c|c|c|c|}
\hline \multirow{2}{*}{ Distribución } & \multicolumn{3}{|c|}{ Lima } & \multicolumn{2}{c|}{ Resto Costa } & \multicolumn{2}{c|}{ Sierra } & \multicolumn{2}{c|}{ Selva } \\
\cline { 2 - 9 } & $\%$ & Total & $\%$ & Total & $\%$ & Total & $\%$ & Total \\
\hline Hasta 3 horas & 3.2 & 144 & 0.0 & 0 & 0.0 & 0 & 3.3 & 9 \\
\hline 4 horas & 3.2 & 144 & 28.6 & 202 & 20.0 & 420 & 6.7 & 19 \\
\hline 5 horas & 38.1 & 1733 & 42.9 & 303 & 36.7 & 770 & 23.3 & 66 \\
\hline 6 ó más horas & 55.6 & 2527 & 28.6 & 202 & 43.3 & 910 & 66.7 & 188 \\
\hline Total & 100.0 & 4549 & 100.0 & 706 & 100.0 & 2100 & 100.0 & 282 \\
\hline
\end{tabular}

Fuente: Encuesta de Gestión a Junta Directivas de Comedores Populares

\section{Cuadro $N^{\circ} 7$ : Estimación del Financiamiento Mensual por el Trabajo Efectuado} por las Socias en los Comedores por Dominio. (en nuevos soles)

\begin{tabular}{|c|c|c|c|c|c|}
\hline Dedicación & Lima & R. Costa & Sierra & Selva & Total \\
\hline Hasta 3 horas & 78,228 & 0 & 0 & 3,478 & 81,706 \\
\hline 4 horas & 104,305 & 93,659 & 248,940 & 9,274 & 456,178 \\
\hline 5 horas & $1,564,569$ & 175,611 & 570,488 & 40,575 & $2,351,243$ \\
\hline 6 ó más horas & $3,650,662$ & 187,318 & $1,078,742$ & 185,487 & $5,102,208$ \\
\hline Total & $5,397,764$ & 456,587 & $1,898,171$ & 238,814 & $7,991,336$ \\
\hline & & \multicolumn{5}{|c|}{ Total Anual } & $95,896,032$ \\
\hline
\end{tabular}

Otra fuente de financiamiento del Programa son las actividades que realizan las socias de los Comedores Populares para atender a los casos sociales. Ya habíamos referido que estos usuarios reciben la ración (el menú) gratuito y como se ve en el Cuadro $\mathrm{N}^{\circ} 8$, en cerca del $20 \%$ de comedores del país se realizan actividades para financiar la atención de estos casos. La actividad que más se realiza es la pollada'. (ver Cuadro $\mathrm{N}^{\circ}$ 9).

Cuadro $N^{\circ} 8$ : Porcentaje de Comedores que Realizaron Actividades para Financiar Casos Sociales por Dominios

\begin{tabular}{|c|c|c|c|c|c|}
\hline \multirow{2}{*}{$\begin{array}{c}\text { JD que } \\
\text { realizan } \\
\text { actividades }\end{array}$} & Nacional & Lima & R. Costa & Sierra & Selva \\
\cline { 2 - 6 } & $\%$ & $\%$ & $\%$ & $\%$ & $\%$ \\
\hline Si & 19.8 & 25.4 & 14.3 & 21.7 & 13.3 \\
\hline No & 80.2 & 74.6 & 85.7 & 78.3 & 86.7 \\
\hline Total & 100.0 & 100.0 & 100.0 & 100.0 & 100.0 \\
\hline
\end{tabular}

Fuente: Encuesta de Gestión a Junta Directivas de Comedores Populares 
César A. Sanabria Montañez

\section{Cuadro No $^{\circ}$ : Principales Actividades que Realizan los Comedores para Financiar la Atención de Casos Sociales 1/}

\begin{tabular}{|c|c|c|c|c|c|}
\hline \multirow{2}{*}{ Actividades } & Nacional & Lima & R. Costa & Sierra & Selva \\
\cline { 2 - 6 } & $\%$ & $\%$ & $\%$ & $\%$ & $\%$ \\
\hline Rifas & 2.5 & 6.3 & 0.0 & 0.0 & 0.0 \\
\hline Polladas * & 95.0 & 100.0 & 100.0 & 100.0 & 50.0 \\
\hline Actividades deportivas & 2.5 & 0.0 & 0.0 & 0.0 & 25.0 \\
\hline Actividades recreativas & 2.5 & 0.0 & 0.0 & 0.0 & 25.0 \\
\hline
\end{tabular}

1/ Incluye solo a los comedores populares cuyas dirigentes respondieron que realizan actividades.

(*) En el termino polladas se incluyen truchadas, parrilladas, cuyadas, frejoladas, etc.

Fuente: Encuesta de Gestión a Junta Directivas de Comedores Populares.

\section{Balance}

En resumen, existen 4 fuentes de financiamiento del Programa de Comedores Populares: el financiamiento público, el pago de bolsillo que realizan los usuarios, el aporte con trabajo que realizan las socias y las actividades que ellas realizan para atender los casos sociales.

En el Cuadro $\mathrm{N}^{\circ} 10$, se muestran los montos de cada fuente para el año 2004 (no incluye el financiamiento de actividades que realizan las socias para los casos sociales). Como se ve, el financiamiento requerido por el Programa para dicho año fue de 284.824.200 nuevos soles, donde las socias y otros usuarios aportaron el $73.6 \%$ del total del financiamiento.

\section{Cuadro No 10 : Financiamiento del Programa de Comedores Populares} por tipo de Fuente. Año 2004 1/ (en nuevos soles )

\begin{tabular}{|l|r|c|}
\hline Fuentes & \multicolumn{1}{|c|}{ Total } & Porcentaje \\
\hline Financiamiento Público & 75.092 .891 & 26,4 \\
\hline Pagos de bolsillo socias y otros usuarios & 113.835 .277 & 40,0 \\
\hline Aportes con trabajo de socias & 95.896 .032 & 33,6 \\
\hline Total & 284.824 .200 & 100,0 \\
\hline
\end{tabular}

$1 /$ No se incluye las actividades que realizan las socias del Comedor para atender los casos sociales.

Como el Estado sólo financia un $26 \%$ del costo del Programa, cabría la posibilidad de que este se concentre más en zonas rurales y se vaya dejando paulatinamente las zonas urbanas de grandes ciudades, donde los comedores podrían autofinanciarse, de este modo concentrar esfuerzos en una mejora en los sectores más pobres del Perú; asimismo, ante el proceso descentralizador, el PRONAA debería enfocar sus esfuerzos hacia la rectoría y apoyo técnico a los gobiernos locales. 
Financiamiento de programas sociales: el caso del programa de comedores populares en el Perú

\section{NOTAS REFERENCIALES}

1. PRONAA: Plan Operativo Institucional Reformulado Unidad Ejecutora 005 PRONAA. Año 2005

2. Ver MIMDES - PRONAA: Evaluación Anual del Plan de Trabajo Institucional-PTI- 2003

3. Información de la Página web del PRONAA http://www.pronaa.gob.pe/a diciembre de 2005.

4. La Comisión Técnica fue constituida el día 19 de setiembre de 2005.

5. Existe un mínimo de atención gratuita a población en caso de abandono, enfermedad, edad avanzada, se señala en el Reglamento para Comedores.

6. Atiende a los beneficiarios de Lima y el Callao.

7. Reglamento para Comedores que reciben apoyo de "Programas de Complementación Alimentaria". Artículo $8^{\circ}$, inciso $\mathrm{k}$.

8. En el estudio se destaca que en promedio a nivel nacional se preparan 68.8 raciones diarias, de las cuales se distribuyen de modo gratuito 2,3 raciones para la Junta Directiva (se entiende que hacen gestiones para la marcha del Comedor), 8 raciones para las socias que participan en la preparación de las raciones y 5,8 son los llamados casos sociales (personas que sufren de discapacidad, enfermedad o abandono social). Entonces se venden el 77,0 $\%$ de las raciones preparadas, distribuidas en $66,0 \%$ para las socias y $11.0 \%$ para las no socias que pagan por la ración. Estos porcentajes se aplicaron para estimar el aporte de bolsillo de los intervenidos de los comedores.

9. La Remuneración Mínima Vital ascendía en el año 2004 a la suma de S/. 460.00 mensuales ó S/.15.33 diarios, según sea el caso; de acuerdo a lo establecido por el Decreto de Urgencia Nº 022-2003 (13/09/2003).

10. La pollada no es otra cosa que pollo a la olla con papas sancochadas y ají molido acompañado de cerveza y jarana. Las parrilladas, anticuchadas y picaronadas, populares en Lima, se han convertido en un recurso de supervivencia frente a la crisis económica y a la falta de empleo en el país. (Tomado de Las polladas: entre el recurso y la fiesta, por Edith Müller E. en: http://www.idl.org.pe/idlrev/revistas/120/pag70.htm )

\section{BIBLIOGRAFÍA}

CEPAL: Gestión de Programas Sociales. En América Latina. Volumen I. Serie Políticas Sociales 25. Santiago de Chile, 1998

Cohen Ernesto y otros: Gestión de programas sociales en América Latina. Análisis de casos Vol. III : El Programa de Restaurantes Escolares Comunitarios de Medellín, Colombia. CEPAL Serie Políticas Sociales 46. Santiago de Chile, marzo de 2001.

Cohen Ernesto, Martínez Rodrigo y Navarrete Cecilia: Gestión de programas sociales en América Latina. Análisis de casos. Vol. I: Proyecto Joven de Argentina. SERIE políticas sociales 46. Santigo de Chile, febrero de 2001

Contreras Eduardo: Evaluación social de inversiones públicas: enfoques alternativos y su aplicabilidad para Latinoamérica. CEPAL. Serie Manuales 37. Santiago de Chile, diciembre del 2004

Fondo de Población de Naciones Unidas: Metodología para Evaluar la Capacidad De Ejecución, en Hallazgo de Evaluación. Número 29 - Marzo de 2000.

Medina Giopp Alejandro: La eficiencia en los programas sociales: análisis de situaciones y propuestas para su mejoramiento. VII Congreso Internacional del CLAD sobre la Reforma del Estado y de la Administración Pública, Lisboa, Portugal, 8-11 Oct. 2002.

MIMDES - Comisión Sectorial de Transferencia: Plan de Transferencia de los Programas Sociales 2003 del Sector MIMDES. Lima - 2003.

Ministerio de Hacienda del Gobierno de Chile: Metodología Evaluación de Impacto. Santiago de Chile, Diciembre de 2004.

Molina Carlos Gerardo: La entrega de los servicios sociales -modalidades y cambios recientes en América Latina-. INDES-BID. Junio, 1999. 